\title{
Cooperative crosslink (un)binding in slowly driven bundles of semiflexible filaments
}

\author{
Claus Heussinger ${ }^{1,2}$ \\ ${ }^{1}$ Institute for Theoretical Physics, Georg-August University of Göttingen, Friedrich-Hund Platz 1, 37077 Göttingen \\ ${ }^{2}$ Max-Planck Institute for Dynamics and Self-Organization, Bunsenstr 10, 37073 Göttingen
}

(Dated: November 15, 2018)

\begin{abstract}
Combining simulations and theory I study the interplay between bundle elastic degrees of freedom and crosslink binding propensity. By slowly driving bundles into a deformed configuration, and depending on the mechanical stiffness of the crosslinking agent, the binding affinity is shown to display a sudden and discontinuous drop. This indicates a cooperative unbinding process that involves the crossing of a free-energy barrier. Choosing the proper crosslinker, therefore, not only allows to change the composite elastic properties of the bundle, but also the relevant time-scales, which can be tuned from the single crosslink binding rate to the much longer escape time over the free-energy barrier.
\end{abstract}

PACS numbers: 87.16.Ka,87.15.Fh,62.20.F-

Filamentous biopolymers, like F-actin or microtubules, play key roles in many cellular and extra-cellular processes. A variety of different crosslinking proteins are used in order to assemble these filaments into higher order structures, like bundles or networks. Permanently crosslinked reconstituted F-actin networks have been used as simple model systems to advance the understanding of the mechanics of the more complex physiological systems [1 3]. Similarly, experiments with actin bundles allowed to assess the influence of the crosslink stiffness on the bundle mechanical properties [4]. A theoretical description was developed that characterizes bundle mechanics starting from the assumption that crosslinks are permanently bound [5]. Recent experiments [6, 7] indicate, however, that low frequency rheology of actin networks can only be understood by properly accounting for crosslink binding and unbinding processes [8]. This points the way towards investigating the possible coupling between filament elasticity and crosslink binding. Several theoretical studies dealt with the equilibrium phase behavior of crosslinked networks [9]. Much less is known for the case of filament bundles. Refs. 10, 11] described an equilibrium unbundling transition in terms of a competition between the entropy of filament bending fluctuations and the attractive force mediated by crosslink binding. Similarly, Ref. [12] invoked filament twist elasticity to explain the apparent thermodynamic stability of bundles with a characteristic radius [13, 14].

Going beyond unconstrained thermal equilibrium, I study here the nonlinear response of a reversibly crosslinked filament bundle to a driving force or deformation. In the crowded environment of a cell F-actin bundles are likely to be constantly exposed to external forces and obstacles that bend, compress or otherwise deform it. Little is known about how the bundle, and in particular the crosslinks inside the bundle, react to such a perturbation. I will show that bundle deformation leads to crosslink unbinding processes that crucially depend on the stiffness of the crosslinking protein. In particular, bundles with stiff crosslinks display a cooperative, discontinuous transition from a highly to a weakly crosslinked state.

Consider a bundle with $N_{f}$ filaments in two spatial dimensions. The bundle is slowly driven into a bent state characterized by the tangent angle $\theta(s)$ at arclength position $s=0 \ldots L$ along the bundle backbone (see Fig. 11). The driving is assumed slow enough such that the crosslink binding degrees of freedom can equilibrate under the constraint of the given bending amplitude.

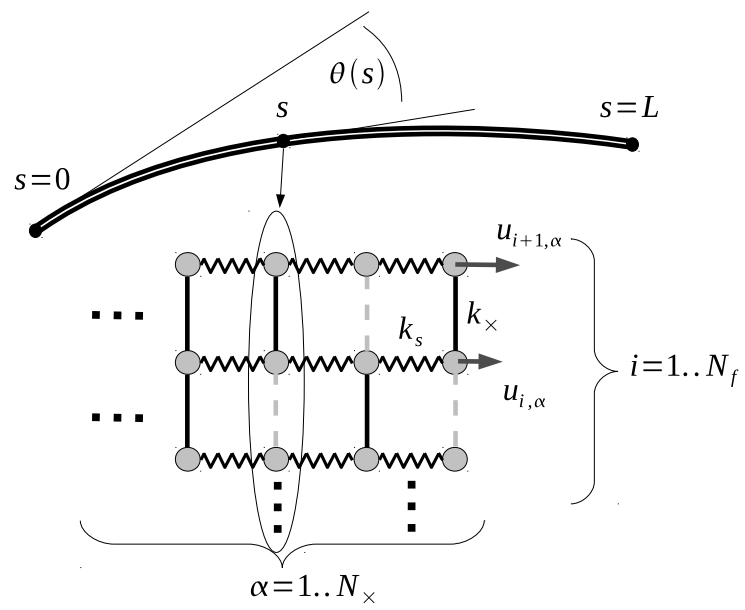

FIG. 1: Illustration of the bundle geometry. The bundle is bent by an arclength-dependent tangent angle $\theta(s)$. Filaments are discretized into beads, which can move along the bundle axis (as quantified by the displacement $u_{i \alpha}$ ) but not in lateral direction. In addition, each bead can host a crosslink that connects it to its neighboring site on the adjacent filament (vertical solid lines: bound crosslink; dashed lines: unbound crosslink).

As a consequence of bundle bending, filaments have to slip relative to each other, thus bringing the crosslinking sites out of registry and leading to crosslink deformation. Denote by $u_{i \alpha}$ the axial slip motion of filament $i$ at ar- 

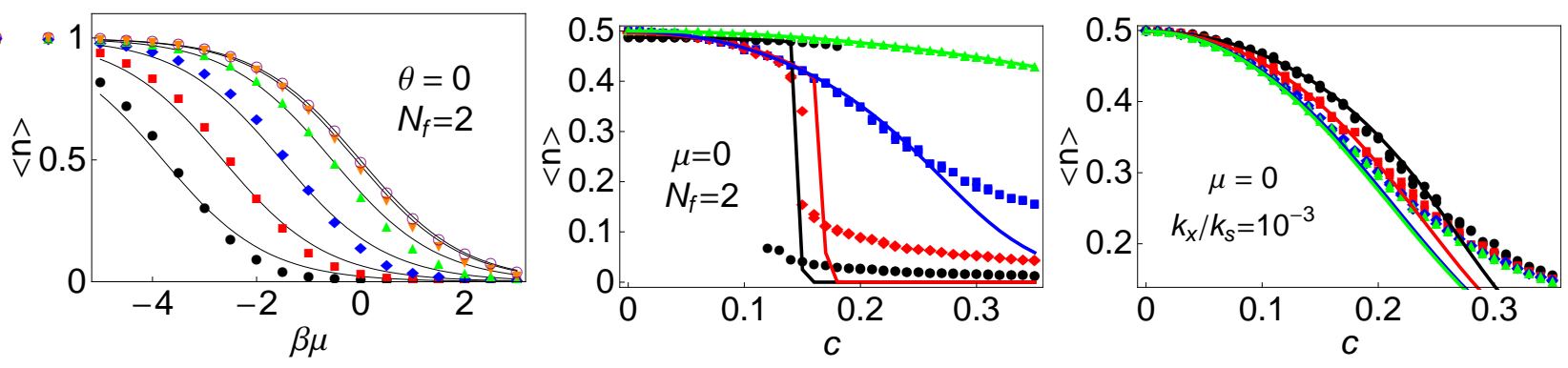

FIG. 2: Average crosslink occupation as function of the control parameters $\mu, k_{\times}, c$ and $N_{f}$ using $N_{\times}=100$ and $\beta k_{s} b^{2}=100$. (left) Undeformed bundle $(\theta \equiv 0)$ for different crosslink stiffness $k_{\times} / k_{s}=10^{-2}, \ldots, 10^{3}$ (from right to left). The solid lines are the solutions of the one-crosslink model, Eq. (5). (center,right) As function of bundle deformation for different crosslink stiffness, $k_{\times} / k_{s}=10^{-5} \ldots 10^{-2}$ (center) and for different filament numbers $N_{f}=2 \ldots 8$ (right). For the largest crosslink stiffness (black circles, center) the simulations display considerable hysteresis, indicating a strongly first-order transition, where a free-energy barrier does not permit to sample efficiently. The lines are obtained without fit parameters from the theoretical model, Eq. (7).

clength position $s_{\alpha}$ (Fig. 1), then the crosslink (shear) deformation can be taken as $\Delta_{i \alpha}=u_{i+1 \alpha}-u_{i \alpha}+b \theta\left(s_{\alpha}\right)$, where $b$ is the lateral distance between the two filaments that are connected by the crosslink. In the continuum limit this reduces to the well known expression for the shear strain, $\partial u_{x} / \partial y+\partial u_{y} / \partial x$, with the axial $\left(u_{x}\right)$ and the lateral $\left(u_{y} \sim \int_{s} \theta\right)$ components of the displacement field. Bringing the two crosslinking sites back into registry $(\Delta \rightarrow 0)$ is therefore only possible if one of the filaments stretches out farther than its connected partner, $u_{i+1 \alpha}=u_{i \alpha}+b \theta\left(s_{\alpha}\right)$, in order to compensate for the bending induced mismatch, $b \theta$. This leads to the competition between filament stretching and crosslink shearing as a fundamental mechanism governing bundle mechanics [15]: Filament stretching can only be relieved at the cost of crosslink shearing; alternatively, if crosslinks remain unsheared, the filaments automatically have to stretch out.

By discretizing the filaments at the sites of the crosslinks one can write the stretching and the shearing energy as a sum over all filaments and all crosslinking sites as

$$
\begin{aligned}
& H_{s}=\frac{k_{s}}{2} \sum_{i=1}^{N_{f}} \sum_{\alpha=1}^{N_{\times}}\left(u_{i \alpha+1}-u_{i \alpha}\right)^{2} \\
& H_{\times}=\frac{k_{\times}}{2} \sum_{i=1}^{N_{f}-1} \sum_{\alpha=1}^{N_{\times}} n_{i \alpha}\left(u_{i+1 \alpha}-u_{i \alpha}+b \theta_{\alpha}\right)^{2}
\end{aligned}
$$

Filaments are thus characterized by a stretching stiffness, $k_{s}$ that constrains the axial motion of two successive sites along a given filament $i$. Crosslinks with stiffness $k_{\times}$couple two filaments laterally, but at the same arclength position, $\alpha$. This energy expression, which has been given previously [5, 15], simply corresponds to a discretized version of anisotropic continuum elasticity [16]. The important new ingredient is the possibility of crosslink (un)binding, which is accounted for by introducing the occupation variables, $n_{i \alpha}=0,1$, corresponding to the unbound and the bound state, respectively.

Assuming $\theta(s)$ to be constant on the time-scales of interest, the bending energy of the bundle needs not be considered explicitly, as it contributes an unimportant constant. The degrees of freedom of the bundle are then the axial displacements $u_{i \alpha}$ of the sites, as well as the occupation with crosslinks, $n_{i \alpha}$. I neglect the possibility of individual filaments performing lateral motion transverse to the bundle axis. It has been shown [10, 11] that at sufficiently low crosslink concentration the entropy gained by these fluctuations can drive an unbundling transition. Being primarily interested in highly crosslinked bundles away from the unbundling transition, these degrees of freedom can be assumed to be frozen out 21].

This ends the model definition. We will now proceed to calculate the average crosslink occupation $\langle n\rangle$ as a function of the control parameters. Combining Metropolis Monte-Carlo simulations with theoretical analysis it will be possible to elucidate the complex interrelationship between crosslink binding and bundle elasticity.

For the simulations, units are chosen such that $b=$ $k_{s}=1$. The binding variables $n_{i \alpha}$ enter Eq. (2) linearly, so they can be traced out exactly [11]. As a result one obtains an effective crosslink potential that only depends on the axial displacements $u_{i \alpha}$

$$
V_{\times}^{\mathrm{eff}}=-k_{B} T \sum_{i \alpha} \log \left(1+e^{-\beta \mu} \exp \left(-\frac{\beta k_{\times}}{2} \Delta_{i \alpha}^{2}\right)\right) .
$$

The depth of the potential is given by $\beta V(0)=\log (1+$ $\left.e^{-\beta \mu}\right)$ and corresponds to the standard free energy of a single crosslink with chemical potential $\mu$. The potential range is set by the crosslink stiffness, $\Delta_{\max } \sim 1 / \sqrt{\beta k_{\times}}$.

Fig. 2 a displays the resulting crosslink occupation $\langle n\rangle$ for an undeformed bundle $(\theta \equiv 0)$ as a function of $\mu$ and $k_{\times}$. It comes as no surprise that crosslinks tend to unbind by either reducing the potential depth $(\mu \nearrow)$ or 
decreasing its range $\left(k_{\times} \nearrow\right)$. Accordingly, already a simple toy-bundle with only one crosslink and two $u$-degrees of freedom allows to reproduce the simulated curves fairly well. The Hamiltonian of this simple model reads

$$
H=\frac{k_{s}}{2}\left(u_{1}^{2}+u_{2}^{2}\right)+\frac{n k_{\times}}{2}\left(u_{1}-u_{2}+b \theta\right)^{2}+\mu n,
$$

and one obtains for the crosslink occupation $(\theta=0)$

$$
\langle n\rangle=\left(1+e^{\beta \mu} \sqrt{1+\frac{2 k_{\times}}{k_{s}}}\right)^{-1}
$$

which is shown together with the Monte-Carlo data in Fig. 2a. The decreasing crosslink occupation is driven by the interplay of binding energy, as characterized by $\mu$, and the configurational entropy stored in the axial displacement modes. Notably, there is no sudden unbundling transition as in the models of Refs. [10, 11]. In those systems, it is the entropy stored in the transverse bending fluctuations that drives the transition towards the unbundled state.

Let us now discuss the crosslink occupation as a function of bundle deformation. For specifity, I take $\theta(s)=$ $c N_{\times} \sin (q s)$ with $q=\pi / 2 L$, corresponding to an approximate solution for a clamped bundle that is loaded at its free end. The parameter $c$ reflects the curvature of the bundle, $c \sim \theta^{\prime}$, and will serve in the following as a measure of the amplitude of the imposed deformation.

As is evident from Fig. 2b,c the crosslink occupation $\langle n\rangle$ decreases upon increasing bundle deformation $c$. This agrees well with the above mentioned notion of bendinginduced mismatch between the binding sites. An increasing mismatch implies increasing strain on the crosslink, which, in turn, increases their tendency to unbind. Unlike the case discussed in Fig. 2a, however, the decrease of $\langle n\rangle$ is gradual and smooth only below a certain value of the crosslink stiffness $k_{\times}$. Above this value, $\langle n\rangle$ displays a very sudden and discontinuous drop with bundle deformation. Interestingly, this effect cannot be explained on the basis of the one-crosslink toy-model of Eq. (4), which only gives a smooth decay governed by a renormalized chemical potential

$$
\tilde{\mu}=\mu+\frac{(b \theta)^{2}}{2} \cdot \frac{k_{s} / 2}{1+k_{s} / 2 k_{\times}}
$$

One concludes, that the discontinuous transition represents a truly cooperative effect, where many crosslink unbinding events are needed to drive the bundle into a weakly crosslinked state.

Analytical progress can be made in a mean-field approximation. To this end we go back to the original Hamiltonian of Eq. (2) and substitute the actual crosslink state $n_{i \alpha}$ by its bundle averaged value, $n=$ $\sum_{i \alpha} n_{i \alpha} /\left(N_{f}-1\right) N_{\times}$. As compared to the fully occupied bundle $(n=1)$, this has the effect of renormalizing the crosslink stiffness $k_{\times} \rightarrow n k_{\times}$.
The advantage of this approximation is that the $u$ variables can now be integrated out by going to the continuum limit and transforming to Fourier space. The resulting effective free energy (per crosslink), which still depends on the bundle averaged occupation variable $n$ has the rather simple structure 22]

$$
\begin{aligned}
F_{\text {eff }}(n) & =\frac{A}{1+n_{0} / n}+\mu n \\
& +k_{B} T[n \log (n)+(1-n) \log (1-n)],
\end{aligned}
$$

Next to the chemical potential $\mu$, there are two relevant parameters. The crosslink stiffness is encoded in $n_{0} \simeq\left(k_{s} / k_{\times}\right) N_{f}\left(N_{f}+1\right) / N_{\times}^{2}$, while the parameter $A$ is related to the amplitude of the bending deformation, $A \simeq k_{s} b^{2} c^{2} N_{f}\left(N_{f}+1\right)$. The term with the logarithm corresponds to the standard entropy of mixing and counts the number of states that are compatible with a given average crosslink occupation. Calculating the average $\langle n\rangle$ from Eq. (77) can easily be performed numerically, and is plotted as solid lines in Fig 2b,c. As can be seen the model and the simulation are in good agreement without fit parameters.

At large bending amplitudes some deviations are apparent. By looking more closely into the distribution of crosslinks inside the bundle one finds that this state is actually highly inhomogeneous, with many more crosslinks bound close to the clamped end than at the free end. In fact, the discontinuous reduction of bound crosslinks proceeds (with our MC-"dynamics") via an unzipping mechanism, where the crosslinks at the free end unbind first. An unbinding front then moves rapidly towards the clamped end of the bundle where it stabilizes some distance away. A similar inhomogeneity can occur in the lateral direction, with crosslinks on different filaments unbinding at different bending amplitudes, leading to a sequence of discontinuous transitions, each corresponding to the unbinding of a single filament pair. I leave the detailed discussion of these complex unbinding pathways for a future publication.

More insight into the physical mechanism that leads to the observed discontinuous transition can be obtained with the help of a saddle-point analysis. By minimizing Eq. (7) with respect to $n, d F_{\text {eff }} / d n=0$, one obtains the phase diagram depicted in Fig. 3. At $T=0$ (left figure), i.e. without the entrop of mixing, the model has two minima at $\langle n\rangle=0,1$ that coexist in a certain range of parameter values (limited by the dashed black lines). The condition $F_{\text {eff }}(0)=F_{\text {eff }}(1)$ then gives a line of discontinuous transitions (solid red line) where the crosslink occupation jumps from $\langle n\rangle=1$ to $\langle n\rangle=0$. For large crosslink stiffness, the bending amplitude at the transition depends on the number of filaments as $c_{\infty} \simeq\left(N_{f}\left(N_{f}+1\right)\right)^{-1 / 2}$ (vertical dashed line).

At finite temperatures (right figure) this line terminates at a critical point, beyond which the crosslink oc- 


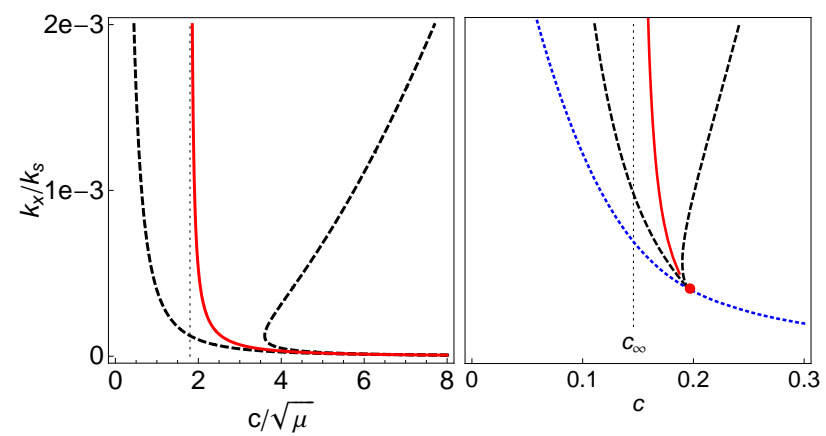

FIG. 3: Phase-diagram from a saddle-point analysis of Eq. (7) at $T=0$ (left) and for finite temperatures (right). In the latter case, the discontinuous transition (solid red line) terminates at a critical point, which shifts with the chemical potential along the (dotted) blue line. Metastable states exist within region delineated by (dashed) black lines. In the limit of large crosslink stiffness $k_{\times} \rightarrow \infty$ (thin vertical line) the discontinuous transition asymptotes at $c_{\infty} \sim 1 / \sqrt{N_{f}\left(N_{f}+1\right)}$.

cupation decreases smoothly with increasing bending amplitude. The critical point itself depends on the chemical potential $\mu$ as indicated in the figure by the (dotted) blue line. Thus, stiff crosslinks tend to unbind cooperatively in a discontinuous transition, while soft crosslinks unbind one after the other leading to a smooth decrease of the average crosslink occupation.

This qualitative difference between bundles with soft or stiff crosslinks can be intuitively understood as follows. When the crosslinks are soft, they cannot induce any stretching in the filaments. Accordingly the force in the crosslinks is small and set by the crosslink stiffness $k_{\times}$. In this limit unbinding events are purely local with no consequences for the rest of the bundle. In contrast, stiff crosslinks are able to drive the filaments into a stretched configuration. The force in the crosslink is now set by the filament stretching stiffness $k_{s}$. An unbinding event will then affect the force balance in the filaments, with the potential of influencing the bundle state far away from the unbinding site. Note, that a similar distinction between soft and stiff crosslinks has been observed in Ref. [17], dealing with the phase behavior of helical filament bundles.

The discontinuous transition and the associated hysteresis observed in the simulations (see Fig 20 b) is tantamount to a long time-scale associated with the escape over a free-energy barrier that separates highly crosslinked from weakly crosslinked states. Thus, the choice of crosslinker not only determines the mechanical properties of a bundle but also affects the relevant relaxation times. Depending on the stiffness of the crosslink, this time-scale may range from the single crosslink binding rate to the escape time over the free-energy barrier.

Such a long time-scale may find its analogy in a recent experiment with $\mathrm{F}$-actin bundles crosslinked by $\alpha$ - actinin [18]. In the experiment a bundle was released from a bent configuration after keeping it there for a certain waiting time. If the waiting time was long enough, the bundle did not relax back into the expected straight state, but remained with a considerable residual bending deformation. It seems that under bundle deformation new binding sites become available that stabilize the bent shape by allowing the crosslinks to rebind in a "native", unstrained state. Interestingly, the apparent time-scale (the waiting time) necessary to observe residual bundle bending was considerably longer than the time required for (un)binding of the individual $\alpha$-actinin linker. By taking $\mu=0$ one can set up a simple correspondence between unbinding events as defined in the present work, and rebinding events into the new sites in the experiment. With this mapping the long time-scale observed in the experiment can be identified with the escape time over the free energy barrier, which is estimated as $\tau \approx 2^{N_{\text {tot }}} / N_{\text {tot }}$, where $N_{\text {tot }}=\left(N_{f}-1\right) N_{\times}$is the total number of binding sites [19, 20]. In this spirit, one would call $\alpha$-actinin, the crosslinker used in the experiment, a stiff crosslink. It would be interesting to repeat the experiment with a softer crosslink. Future experiments should furthermore measure the force needed to bend the bundle, as this is expected to scale with crosslink occupation and display a discontinuous drop, $\Delta F \sim N_{f}^{2}[20]$. In particular, it would be interesting to discuss rate-dependent bundle bending. This would be a natural next step in the effort to understand the full dynamics of the coupled processes of bundle conformation and crosslink binding. Finally, it should be pointed out that crosslinked actin networks typically consist of composite mixtures of single filaments and small bundles [2]. Therefore, the observed unbinding transition could directly be relevant for F-actin network rheological properties.

Fruitful discussions with M. Bathe, E. Frey, D. Strehle and R. Vink are gratefully acknowledged.

[1] A. Bausch and K. Kroy, Nature Physics 2, 231 (2006).

[2] O. Lieleg, M. M. A. E. Claessens, and A. R. Bausch, Soft Matter 6, 218 (2010).

[3] K. E. Kasza et al., Curr. Opin. Cell Biol. 19, 101 (2007).

[4] M. M. A. E. Claessens, M. Bathe, E. Frey, and A. R. Bausch, Nature Mat. 5, 748 (2006).

[5] M. Bathe et al., Biophys. J. 94, 2955 (2008).

[6] O. Lieleg, M. M. A. E. Claessens, Y. Luan, and A. R. Bausch, Phys. Rev. Lett. 101, 108101 (2008).

[7] C. P. Broedersz et al., arXiv:1006.3940 (2010).

[8] L. Wolff, P. Fernandez, and K. Kroy, arXiv:1001.0907 (2010).

[9] A. G. Zilman and S. A. Safran, Europhys. Lett. 63, 139 (2003). I. Borukhov, R. F. Bruinsma, W. M. Gelbart, and A. J. Liu, PNAS 102, 3673 (2005). P. Benetatos and A. Zippelius, Phys. Rev. Lett. 99, 198301 (2007).

[10] P. Benetatos and E. Frey, Phys. Rev. E 67, 051108 
(2003).

[11] J. Kierfeld, T. Kühne, and R. Lipowsky, Phys. Rev. Lett. 95, 038102 (2005).

[12] G. Grason and R. Bruinsma, Phys. Rev. Lett. 99, 098101 (2007).

[13] O. Pelletier et al., Phys Rev Lett 91, 148102 (2003).

[14] M. M. A. E. Claessens et al., Proc. Natl. Acad. Sci. USA 105, 8819 (2008).

[15] C. Heussinger, M. Bathe, and E. Frey, Phys. Rev. Lett. 99, 048101 (2007). C. Heussinger, F. Schüller, and E. Frey, Phys. Rev. E 81, 021904 (2010).

[16] A. E. H. Love, A treatise on the mathematical theory of elasticity, 4th ed., Dover, New York (1944).
[17] H. Shin et al., Phys. Rev. Lett. 103, 238102 (2009).

[18] D. Strehle et al., Eur. Biophys. J. 40, 93 (2011).

[19] T. Erdmann, and U. S. Schwarz, J. Chem. Phys. 121, 8997 (2004).

[20] C. Heussinger, in preparation (2011).

[21] Short wavelength bending fluctuations can also be viewed as being incorporated into an effective stretching stiffness $k_{s}^{\text {eff }}$ which then contains both enthalpic and entropic effects

[22] As for the MC simulations I take $\theta(s) \sim \sin (q s)$ and consider the lowest mode only, $q=\pi / 2 L$. 